\title{
All-Optical Magnetic Recording with Circularly Polarized Light
}

\author{
C. D. Stanciu, ${ }^{1, *}$ F. Hansteen, ${ }^{1}$ A. V. Kimel, ${ }^{1}$ A. Kirilyuk, ${ }^{1}$ A. Tsukamoto, ${ }^{2}$ A. Itoh, ${ }^{2}$ and Th. Rasing ${ }^{1}$ \\ ${ }^{1}$ Institute for Molecules and Materials, Radboud University Nijmegen, Toernooiveld 1, 6525 ED Nijmegen, The Netherlands \\ ${ }^{2}$ College of Science and Technology, Nihon University, 7-24-1 Funabashi, Chiba, Japan
}

(Received 2 March 2007; published 25 July 2007)

\begin{abstract}
We experimentally demonstrate that the magnetization can be reversed in a reproducible manner by a single 40 femtosecond circularly polarized laser pulse, without any applied magnetic field. This optically induced ultrafast magnetization reversal previously believed impossible is the combined result of femtosecond laser heating of the magnetic system to just below the Curie point and circularly polarized light simultaneously acting as a magnetic field. The direction of this opto-magnetic switching is determined only by the helicity of light. This finding reveals an ultrafast and efficient pathway for writing magnetic bits at record-breaking speeds.
\end{abstract}

The ever increasing operation speed of modern electronics as well as the push to increase the capacity of storing information motivates the search for faster approaches to process and magnetically record information [1-11]. In magnetic memory devices, logical bits ("ones" and "zeros") are stored by setting the magnetization vector of individual magnetic domains either "up" or "down." The conventional way to record a bit is to reverse the magnetization $\mathbf{M}$ by applying a magnetic field $\mathbf{H}$ antiparallel to $\mathbf{M}$ [12]. Much faster magnetization reversal can be obtained if the magnetic field is applied perpendicular to $\mathbf{M}$ via a precessional switching [1-5]. However, it was recently concluded that deterministic precessional switching does not take place at times shorter than 2 picoseconds [6]. At this time scale the magnetization was shown to become fractured by the ultrashort and intense magnetic field pulses employed $(\sim 2 \mathrm{ps}$ duration and a strength of $\sim 3$ Tesla), resulting in a random switching behavior.

Optical pulses could serve as an alternative stimulus to trigger magnetization reversal. In theory, circularly polarized light should have the ability to act upon a magnetic system in a way similar to a magnetic field directed parallel to the wave vector of the light via the inverse Faraday effect $[13,14]$. Moreover, right- and left-handed circularly polarized waves should act as magnetic fields of opposite sign. This effect was only recently confirmed experimentally $[9,11]$ and has also been demonstrated to be an efficient tool for coherently controlling the small angle precessional magnetization dynamics in magnetic dielectrics $[10,15]$.

However, magnetization reversal induced by a subpicosecond stimulus, i.e., a true $180^{\circ}$ switching of the magnetization into a stable and oppositely magnetized state, has remained an important fundamental and technological challenge. Further adding to this challenge is the fact that for direct transfer of angular momentum from the light to the magnetic system, the number of photons available in optical experiments is far from enough [16]. Despite the predicted speed limit and shortage of photons, we here demonstrate that a single 40 femtosecond circularly polar- ized laser pulse can cause well-controlled permanent magnetization reversal in materials typically used for data storage. The optical excitation causes an ultrafast heating of the magnetic system which makes it highly susceptible to the magnetic field simultaneously generated by the circularly polarized light pulse. The combination of theses two effects leads to magnetization reversal in a reproducible way. No external magnetic field is required for this opto-magnetic switching, and the stable final state of the magnetization is unambiguously determined by the helicity of the laser pulse.

For our experiments we have chosen $\mathrm{GdFeCo}$, an amorphous ferrimagnetic alloy that is widely used in magnetooptical recording [17] and known for its strong magnetooptical effects [18]. Thus, it possesses the Faraday rotation of $\sim 200$ times higher than that in orthoferrites in which strong inverse Faraday effect has been observed $(10 \mathrm{mT}$ for $1 \mathrm{~mJ} / \mathrm{cm}^{2}$ laser fluence) [9]. Consequently, in GdFeCo, one might expect the light induced effective field of about $2 \mathrm{~T} / \mathrm{mJ} / \mathrm{cm}^{2}$ that may be sufficient for magnetization reversal. Thin films of this alloy usually exhibit strong perpendicular magnetic anisotropy, a square hysteresis loop, and large magnetic domains which are easily observable in a polarizing microscope. Our samples have a typical composition of $\mathrm{Gd}_{22} \mathrm{Fe}_{74.6} \mathrm{Co}_{3.4}$, a saturation magnetization of about $4 \pi M=1000 \mathrm{G}$ at room temperature, and a Curie point $T_{\mathbf{C}}=500 \mathrm{~K}$. They were grown by magnetron sputtering in a multilayer structure: glass/AlTi(10 nm)/ $\mathrm{SiN}(5 \mathrm{~nm}) / \operatorname{GdFeCo}(20 \mathrm{~nm}) / \mathrm{SiN}(60 \mathrm{~nm})$, where the AlTi layer serves as a heat sink and $\mathrm{SiN}$ is used as a buffer and capping layer.

The experiments were performed by placing a sample under a polarizing microscope, where domains with magnetization "up" and "down" could be observed as white and black regions, respectively. To excite the material we used regeneratively amplified pulses from a Ti:sapphire laser at a wavelength of $\lambda=800 \mathrm{~nm}$ and a repetition rate of $1 \mathrm{kHz}$. Each pulse had a Gaussian intensity profile, with a width at half maximum of $40 \mathrm{fs}$. The laser pulses 
were incident normal to the sample surface, so that an effective optically generated magnetic field would be directed along the magnetization. The beam was focused down to a $100 \mu \mathrm{m}$ spot and the laser-induced magnetic changes were studied by recording magneto-optical images of the domain patterns before, during, and after the laser excitation. This is a well-known technique for studying the magnetic material response to ultrashort field pulses $[1,2,6]$. The experiments were performed at room temperature in air.

The effect of polarized laser pulses on the magnetization is most readily demonstrated by slowly sweeping a laser beam across the surface of the sample. Figure 1 shows how three different sweeps with the laser beam right-handed circularly polarized $\left(\sigma^{+}\right)$, linearly polarized $(L)$, and lefthanded $\left(\sigma^{-}\right)$circularly polarized affect the initial domain pattern in dramatically different ways. The region exposed to linearly polarized light is turned into small domains randomly oriented up or down. In striking contrast, the magnetization of the regions exposed to circularly polarized light is completely switched into an up or down state (white or black) determined only by the helicity of the optical excitation.

Despite the very clean switching behavior observed when scanning the laser beam across the sample, a scramble of the magnetization remains near the right end of each scan line, at the point where the laser beam was shut off. To further investigate its origin we focused the laser beam onto a domain wall, as shown in Fig. 2, and acquired magneto-optical images during the pulsed laser excitation for the three different polarizations of the beam $\sigma^{+}, L$, and $\sigma^{-}$. The gray area observed in the center of the laser spot has a contrast in between that of the white and black magnetic regions. This represents a demagnetized state consisting of small domains which are randomly oriented by each excitation event and not sensitive to the

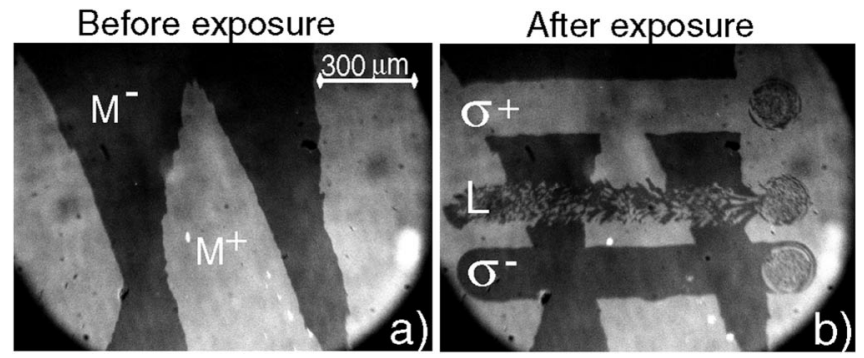

FIG. 1. The effect of ultrashort polarized laser pulses on magnetic domains in $\mathrm{Gd}_{22} \mathrm{Fe}_{74.6} \mathrm{Co}_{3.4}$. (a) Magneto-optical image of the initial magnetic state of the sample before laser exposure. White and black areas correspond to up $\left(M^{+}\right)$and down $\left(M^{-}\right)$magnetic domains, respectively. (b) Domain pattern obtained by sweeping at low speed $(\sim 30 \mu \mathrm{m} / \mathrm{s})$ linear $(L)$, right-handed $\left(\sigma^{+}\right)$, and left-handed $\left(\sigma^{-}\right)$circularly polarized beams across the surface of the sample, with a laser fluence of about $11.4 \mathrm{~mJ} / \mathrm{cm}^{2}$. The central area of the remaining spots at the end of each scan line consists of small magnetic domains, where the ratio of up to down magnetic domains is close to 1 . light polarization. The demagnetized region is formed due to the strong heating in the central part of the Gaussian laser beam. The strongly nonequilibrium electron distribution created by the ultrashort laser excitation leads to a very rapid increase of the spin temperature via an efficient Stoner spin-scattering mechanism [19-24]. An increase of the spin temperature to above the Curie point corresponds to a total demagnetization, and subsequent cooling in zero magnetic field favors the formation of a random multidomain state, as observed in this experiment. Note that the concept of spin temperature used above denotes the amount of energy deposited in to the magnetic system by the laser excitation.

At the outer perimeter of the gray demagnetized area a white (black) ring with perfect contrast can be seen in the case of $\sigma^{+}\left(\sigma^{-}\right)$circularly polarized excitation. When the laser polarization is linear this ringlike switching does not occur, and the demagnetized gray area simply defines the region where the laser fluence is sufficiently high to cause heating above $T_{\mathbf{C}}$. This area has the exact same size for all three polarizations, implying that at the outer perimeter of the laser spot, where the opto-magnetic switching occurs, the temperature always remains below the Curie point. Hence, the laser-induced magnetization reversal can be explained as the result of two cooperating effects: first, part of the pulse energy is absorbed in the metal bringing it into a strongly nonequilibrium state with the spin temperature increasing to a level slightly below the $T_{\mathbf{C}}$. Second, the circularly polarized laser pulse acts on this excited magnetic system as an effective magnetic field directed antiparallel to the small remaining magnetization $[9,10,13,14]$. According to the theory of second-order magnetic phase transitions, the magnetic susceptibility diverges when the spin temperatures approach the Curie point [25]. The observed all-optical magnetization reversal can therefore be seen as the combined result of a diverging magnetic susceptibility and a laser-induced effective magnetic field. This switching process is in some respects similar to the
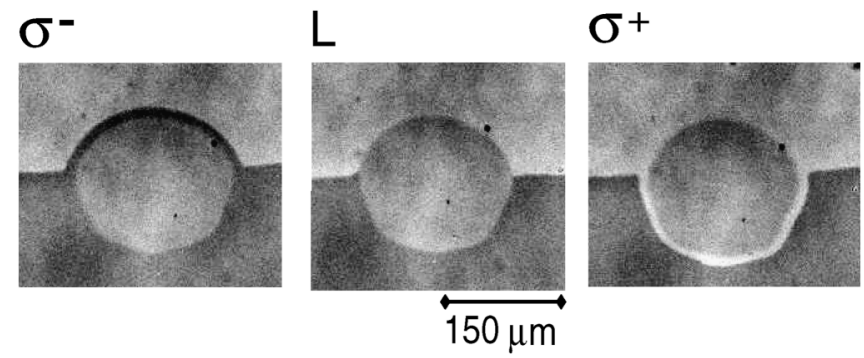

FIG. 2. Images of the laser spot focused onto a domain wall during the $1 \mathrm{kHz}$ pulsed laser excitation with linear $(L)$, right$\left(\sigma^{+}\right)$, and left-handed $\left(\sigma^{-}\right)$circular polarization. The images were obtained for a pulse fluence of about $11.4 \mathrm{~mJ} / \mathrm{cm}^{2}$. In all three cases the central region of the optically excited area is demagnetized (gray color) due to extensive heating. For circularly polarized excitation $\left(\sigma^{+}\right.$and $\left.\sigma^{-}\right)$opto-magnetic switching takes place on the perimeter of the excited area, where the temperature is just below $T_{\mathbf{C}}$. 
recently developed heat assisted magnetic recording scheme [26], but with the major difference that the laser pulse in our case not only heats the medium, but does this ultrafast and simultaneously acts as a magnetic field.

The above described picture suggests that by simply reducing the laser power one should be able to cleanly reverse the magnetization in the center of the excited area without causing overheating and demagnetization. Figure 3 shows how the switching depends on the excitation fluence. The sample was initially magnetized in the up direction and then exposed to laser pulses for about $20 \mathrm{~ms}$. High laser fluences produce similar random multidomain magnetic states for both $\sigma^{+}$and $\sigma^{-}$excitation, while the lower fluence excitations lead to drastically different results for the two helicities. In particular, at $2.9 \mathrm{~mJ} / \mathrm{cm}^{2}$, the $\sigma^{+}$pulses leave the initial magnetic state unchanged, while the $\sigma^{-}$excitation cleanly reverses the magnetization only in the central part of the irradiated area.

Simple estimates show that all-optical switching based on the absorption of the angular momentum of the photons is only feasible at a spin temperature within a range of about $1 \mathrm{mK}$ below $T_{\mathbf{C}}$. However, the $20 \mu \mathrm{m}$ switched spot in Fig. 3 represents a temperature gradient of about $3 \mathrm{~K}$ across the excited area. Consequently, a switching mechanism due to direct transfer of angular momentum via absorption is unlikely. A stimulated Raman scattering mechanism [9] can account for the observed opto-magnetic switching, where the photons efficiently stimulate the transfer of angular momentum from the lattice to the spins, implying that one photon may induce more than one spin flip.

In order to unambiguously determine whether excitation by a single $40 \mathrm{fs}$ laser pulse is sufficient to reverse the magnetization, the laser beam was swept at high speed across the sample, so that each pulse landed at a different spot. The results of this experiment are shown in Fig. 4(a) for the two helicities of the laser excitation. One can see that each of the $\sigma^{+}$pulses reverses the magnetization in the black domain but does not affect the magnetization of the white domain. The opposite situation is observed when the sample is exposed to $\sigma^{-}$pulses. Thus, during the presence of a single $40 \mathrm{fs}$ laser pulse, information about the photons' angular momentum is transferred to the magnetic medium, and subsequently recording occurs. These experiments unambiguously demonstrate that all-optical magnetization reversal can be achieved by single 40 femtosecond circularly polarized laser pulses without the aid of an external magnetic field.

The actual speed of the magnetization reversal is still an intriguing question. It can be answered quite accurately by considering the coherence time of optical excitations in metals. During the $40 \mathrm{fs}$ interaction of the optical pulse with the sample, information about the angular momentum of the photons is transferred to the electrons via electricdipole transitions. However, the electrons lose this information very rapidly, typically within $200 \mathrm{fs}$, due to decoherence processes such as electron-electron and electronphonon collisions [27,28]. Moreover, the optical spectrum of $\mathrm{Gd}_{22} \mathrm{Fe}_{74.6} \mathrm{Co}_{3.4}$ does not show any narrow lines, thus excluding the possibility of any long-lived electronic states acting as a reservoir of angular momentum. It follows that the actual magnetization reversal must take place on a subpicosecond time scale, as this is the only way for the helicity information to survive on longer time scales. Consequently, the magnetization is reduced on the time scale of the laser pulse. This short time scale indicates a direct channel between the electrons and the spin system in $\mathrm{GdFeCo}$. This is an important conclusion since currently, the microscopic spin-flip mechanisms leading to the observed ultrafast reduction of macroscopic magnetization are still a matter of debate $[19,29,30]$. Because the spin temperature of the area where all-optical magnetization reversal occurs remains below the Curie point, some small domains of the magnetically ordered phase are still present [31]. The fact that a circularly polarized laser pulse reverses the magnetization means that the magnetic field

\section{$11.4 \mathrm{~mJ} / \mathrm{cm}^{2}$}
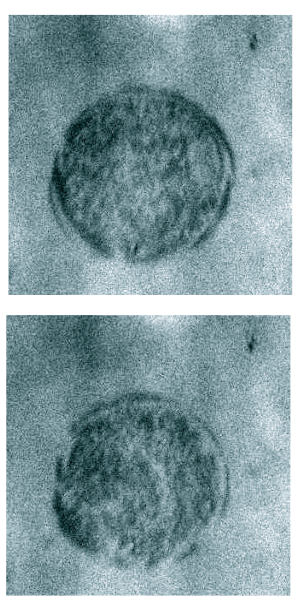
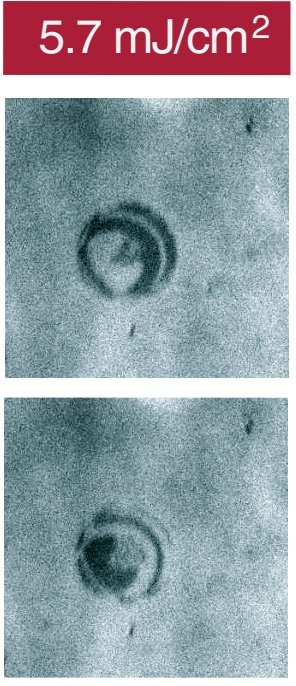
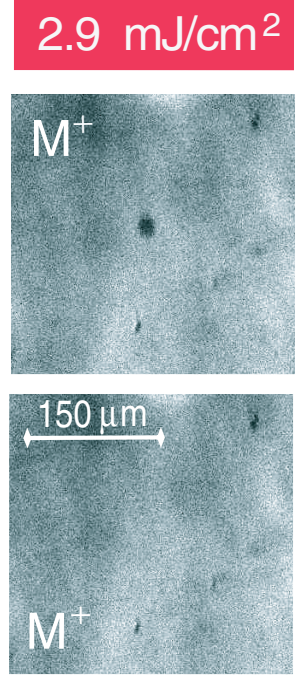

Intensity

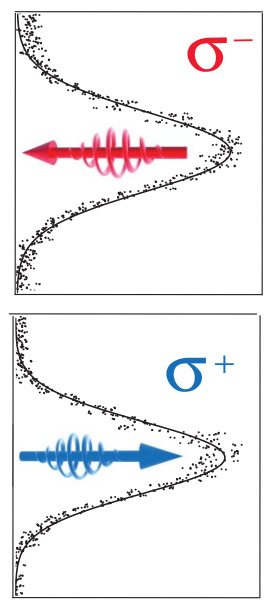

FIG. 3 (color). Images of the magnetic film after exposure to $\sim 20$ ultrashort left-handed $\sigma^{-}$(top row) and righthanded $\sigma^{+}$(bottom row) circularly polarized laser pulses at different pulse energies. Before each exposure, the sample was saturated in the up $\left(M^{+}\right)$state. The graphs on the right show the intensity profile of the laser beams used for excitation. 
a)

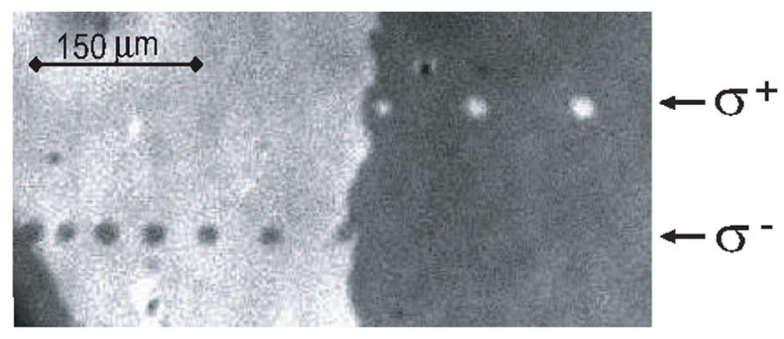

b)

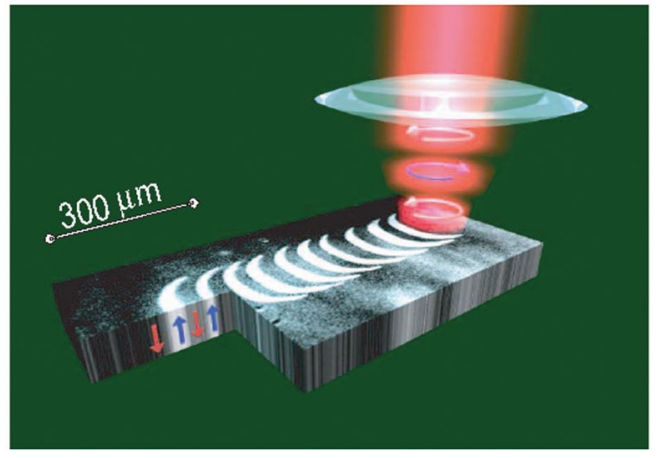

FIG. 4 (color). All-optical magnetic recording by femtosecond laser pulses. (a) The effect of single 40-fs circular polarized laser pulses on the magnetic domains in $\mathrm{Gd}_{22} \mathrm{Fe}_{74.6} \mathrm{Co}_{3.4}$. The domain pattern was obtained by sweeping at high-speed $(\sim 50 \mathrm{~mm} / \mathrm{s})$ circularly polarized beams across the surface so that every single laser pulse landed at a different spot. The laser fluence was about $2.9 \mathrm{~mJ} / \mathrm{cm}^{2}$. The small size variation of the written domains is caused by the pulse-to-pulse fluctuation of the laser intensity. (b) Demonstration of compact all-optical recording of magnetic bits. This was achieved by scanning a circularly polarized laser beam across the sample and simultaneously modulating the polarization of the beam between left and right circular.

generated by the laser is able to reverse the magnetization in these domains. The complete recovery of the magnetization in the new state is a much slower process determined mainly by the speed of heat diffusion in the sample. During this recovery process no magnetic field is present to set the magnetization, but the magnetization direction is well determined.

As a simple illustration of opto-magnetic recording it is shown in Fig. 4(b) how optically written bits can be overlapped and made much smaller than the beam waist by modulating the polarization between $\sigma^{+}$and $\sigma^{-}$as the laser beam is swept across the sample. High density recording may also be achieved by employing especially designed near-field antenna structures [26] such as those currently being developed for heat assisted magnetic recording. With the recent development of compact ultrafast laser systems [32] and the successful incorporation of lasers in magnetic storage devices [26], the present demonstration of ultrafast and all-optical magnetization reversal might spur the realization of a new generation of magnetic recording devices.

In conclusion, we have demonstrated that the magnetization can be reversed in a controllable way with a single 40 fs circularly polarized laser pulse without the aid of any applied magnetic field. The direction of this switching is determined only by the helicity of the light pulse. Beside its clear technological importance, we believe that the observations reported here will lead to a better understanding of the interaction of light with magnetic systems on ultrashort time scales.

We thank T. Toonen, W. Szweryn, and A. van Etteger for the technical support. This work was partially supported by the European network DYNAMICS, NWO, FOM, and the Grant in Aid for Scientific Research of the MEXT in Japan, Grants No. 18560347 and No. 18760239.

*Corresponding author.

d.stanciu@science.ru.nl

[1] C. H. Back et al., Science 285, 864 (1999).

[2] C. H. Back et al., Phys. Rev. Lett. 81, 3251 (1998).

[3] S. Kaka and S.E. Russek, Appl. Phys. Lett. 80, 2958 (2002).

[4] H. W. Schumacher et al., Phys. Rev. Lett. 90, 017201 (2003).

[5] Th. Gerrits et al., Nature (London) 418, 509 (2002).

[6] I. Tudosa et al., Nature (London) 428, 831 (2004).

[7] C. D. Stanciu et al., Phys. Rev. B 73, 220402(R) (2006).

[8] Ganping Ju et al., Phys. Rev. Lett. 93, 197403 (2004).

[9] A. V. Kimel et al., Nature (London) 435, 655 (2005).

[10] F. Hansteen et al., Phys. Rev. Lett. 95, 047402 (2005).

[11] C. D. Stanciu et al., Phys. Rev. Lett. 98, 207401 (2007).

[12] M. Mansuripur, Physical Principles of Magneto-Optical Recording (Cambridge University Press, Cambridge, England, 1998).

[13] L. P. Pitaevskii, Sov. Phys. JETP 12, 1008 (1961).

[14] J.P. van der Ziel, P. S. Pershan, and L.D. Malmstrom, Phys. Rev. Lett. 15, 190 (1965).

[15] A. V. Kimel et al., J. Phys. Condens. Matter 19, 043201 (2007).

[16] B. Koopmans et al., Phys. Rev. Lett. 85, 844 (2000).

[17] H. Awano et al., Appl. Phys. Lett. 69, 4257 (1996).

[18] P. Hansen et al., J. Appl. Phys. 66, 756 (1989).

[19] E. Beaurepaire et al., Phys. Rev. Lett. 76, 4250 (1996).

[20] J. Hohlfeld et al., Phys. Rev. Lett. 78, 4861 (1997).

[21] G. P. Zhang and W. Hübner, Phys. Rev. Lett. 85, 3025 (2000).

[22] L. Guidoni, E. Beaurepaire, and J.-Y. Bigot, Phys. Rev. Lett. 89, 017401 (2002).

[23] J. Hohlfeld et al., Phys. Rev. B 65, 012413 (2001).

[24] A. Scholl et al., Phys. Rev. Lett. 79, 5146 (1997).

[25] L.D. Landau and E. M. Lifshitz, Electrodynamics of Continuous Media (Pergamon, New York, 1984).

[26] W. A. Challener et al., Jpn. J. Appl. Phys. 42, 981 (2003).

[27] H. Petek and S. Ogawa, Prog. Surf. Sci. 56, 239 (1997).

[28] V. V. Kruglyak et al., Phys. Rev. B 71, 233104 (2005).

[29] Uwe Bovensiepen, J. Phys. Condens. Matter 19, 083201 (2007).

[30] M. Cinchetti et al., Phys. Rev. Lett. 97, 177201 (2006).

[31] O. Chubykalo-Fesenko et al., Phys. Rev. B 74, 094436 (2006).

[32] U. Keller, Nature (London) 424, 831 (2003). 